\title{
Predicción de la abundancia y tasa de infección de Triatoma dimidiata: un mapa de riesgo de transmisión natural de la enfermedad de Chagas en la Península de Yucatán, México.*
}

Contribución de la UADY a la Medicina

Eric Dumonteil ${ }^{1}$, Sébastien Gourbière².

${ }^{1}$ Laboratorio de Parasitología, Centro de Investigaciónes Regionales “Dr. Hideyo Noguchi”, Universidad Autónoma de Yucatán, Mérida, Yucatán, México; ${ }^{2}$ Laboratoire de Théorie des Systèmes, Université de Perpignan, France.

\section{RESUMEN.}

La enfermedad de Chagas es un problema de salud pública de mayor importancia en America latina, causado por el parásito protozoario Trypanosoma cruzi y transmitido por insectos hematófagos de la subfamilia Triatominae. El control está basado en el control de los insectos domésticos con insecticidas y con el mejoramiento de los domicilios. Como para otras enfermedades transmitidas por vectores, la identificación de las zonas de alto riesgo de transmisión del parásito es de gran importancia para la planificación e implementación de programas de control eficientes. En este estudio exploramos las relaciones entre la distribución geográfica de Triatoma dimidiata y factores bioclimáticos en la península de Yucatán, México, utilizando sistemás de información geográfica,

*Artículo publicado en Am J Trop Med Hyg 2004; 70:514-9. Reproducido con autorización. y desarrollamos modelos predictivos de la abundancia de T. dimidiata en los domicilios y de su tasa de infección por T. cruzi. Utilizamos estas predicciones para la elaboración del primer mapa de riesgo de transmisión natural de la enfermedad de Chagas en la península de Yucatán, una heramienta de gran utilidad para la implementación de programas de control en la región. (Rev Biomed 2004; 15:221-231)

Palabras clave: Enfermedad de Chagas, sistema de información geográfica, Triatoma dimidiata, Yucatán México.

\section{SUMMARY.}

Prediction of the abundance and rate of infection of Triatoma dimidiata: a map of the natural transmission risk for Chagas disease in the Yucatan Peninsula, Mexico.

Solicitud de sobretiros: Eric Dumonteil, Ph.D. Laboratorio de Parasitología, Centro de Investigaciones Regionales "Dr. Hideyo Noguchi", Universidad Autónoma de Yucatán, Ave. Itzaes \# 490 x 59, C.P. 97000, Mérida, Yucatán, México. Tel: (52-9) 924-5910

Fax: (52-9) 923-6120 E-mail: : oliver@tunku.uady.mx

Recibido el 30/Agosto/2004. Aceptado para publicación el 7/Septiembre/2004.

Este artículo está disponible en http://www.uady.mx/sitios/biomedic/revbiomed/pdf/rb041544.pdf

Vol.15/No.4/Octubre-Diciembre, 2004 


\section{E Dumonteil, S Gourbière.}

Chagas disease is a major public health problem in Latin America, caused by the protozoan parasite Trypanosoma cruzi and transmitted by hematophagous insects from the Triatominae subfamily. Control is based on domestic vector control with insecticides and housing improvement. As with other vector-borne diseases, the identification of areas of high risk of disease transmission is a major prerequisite for the planning and implementation of cost-effective control programs. In this study, we explored the relationship between the geographic distribution of Triatoma dimidiata and bioclimatic factors in the Yucatan peninsula in Mexico, using geographic informational systems and developed predictive models of $T$. dimidiata domestic abundance and of its infection rates by $T$. cruzi. These predictions were then used to build the first natural transmission risk map for Chagas disease in the Yucatan peninsula, a tool that should prove very valuable for the implementation of effective vector control programs in the region. (Rev Biomed 2004; 15:221-231)

Key words: Chagas disease, geographic information system, Triatoma dimidiata, Yucatan Mexico.

La enfermedad de Chagas, o Tripanosomiasis Americana, es causada por el parásito protozoario Trypanosoma cruzi, y transmitida por insectos hematófagos de la subfamilia Triatominae. Esta enfermedad representa un problema de salud pública de primer importancia en America latina, donde la OMS estima que 100 millones de personas están en riesgo de infección, y 16-18 millones están infectados (1). En México, la enfermedad de Chagas es endémica en varias regiones, incluyendo la península de Yucatán, donde se han reportado tasas de seroprevalencia de $11-18 \%$ y $5.6 \%$ en la población general y donantes de sangre, respectivamente (2).

Debido a la baja eficacia de los tratamientos farmacológicos y la ausencia de una vacuna, el control de la enfermedad de Chagas se sigue basando en el control vectorial con insecticidas y con el mejoramiento de los domicilios (1). En Suramérica, estos programas de prevención han sido muy exitosos para reducir las tasas de infestación de los domicilios por triatominos y, en consecuencia, la transmisión natural de la enfermedad de Chagas a humanos. Así, se espera que en los próximos años, la transmisión natural de la enfermedad de Chagas sea interumpida en los países del cono sur (Argentina, Brazil, Bolivia y Chile), mientras que programas similares han sido iniciados en Centro América (3).

En la península de Yucatán, Triatoma dimidiata es el único vector. En un trabajo anterior, reportamos los primeros datos sobre la distribución de $T$. dimidiata en los domicilios de la península de Yucatán y sus variaciones estacionales (4). Demostramos que T. dimidiata era más abundante en los domicilios y su tasa de infección por T. cruzi más alta durante la epoca de calor de Abril-Junio, sugiriendo un mayor riesgo de transmisión de T. cruzi a humanos durante este periodo. Además, este vector de la enfermedad de Chagas era más abundante en la parte norte de la península, posiblemente debido a factores ambientales (4). De hecho, varios otros estudios han demostrado que los factores bioclimáticos pueden influir dramaticamente en la distribución geográfica de los triatominos (5-8). Como en el casos de otras enfermedades transmitidas por vectores, el conocimiento de la distribución geográfica del vector, su capacidad vectorial y, de manera general, las zonas de alto riesgo de transmisión de la enfermedad, es de suma importancia para el desarrollo de herramientas sensibles para la predicción de casos y la optimización de la planeación e implementación de programas de control efectivos.

En años recientes, herramientas analíticas como los sistemás de información geográfica (SIG), la percepción remota (SR) y el modelado de nicho ecológico han ampliando considerablemente las posibilidades de analizar las relaciones entre los factores ambientales y la ecología de los vectores o la transmisión de enfermedades. De hecho, esas herramientas han sido utilizadas extensivamente para definir la distribución geográfica de enfermedades como la malaria (9-13), tripanosomiasis Africana (14, $15)$, esquistosomiasis $(16,17)$, leishmaniasis (18-21),

\section{Revista Biomédica}




\section{Mapa de riesgo de la enfermedad de Chagas.}

o enfermedad de Lyme (22-24), entre otras, a una escala continental o de país. La mayoría de estos estudios se enfocaron a la predicción de la presencia/ ausencia del vector, pero algunos predicen la densidad del vector, su tasa de infección o el riesgo de transmisión de la enfermedad. Estos mapas predictivos han resultado muy útiles para el seguimiento de las enfermedades y su control, y son cada vez más utilizados en salud pública (25-27).

Este tipo de análisis está empezando a ser aplicado a la enfermedad de Chagas, para la cual el control se ha basado principalmente en intensivos estudios de campo para evaluar índices entomológicos y epidemiológicos. Se utilizó recientemente la PR y los SIG para predecir la presencia/ausencia de Triatoma infestans en Sudamérica (28). Este trabajo demostró que el índice de vegetación y 5 factores climáticos son suficientes para predecir la distribución de $T$. infestans a escala continental, y confirmó que este tipo de análisis puede reducir considerablemente los estudios de campo necesarios. En otro trabajo, se usó el modelaje de nichos ecológicos para predecir la distribución geográfica de triatomineos del complejo Protracta y de sus reservorios potenciales en México (29). Este tipo de modelo resultó muy poderoso para el análisis de las relaciones entre los vectores y hospederos, y para la identificación de áreas de riesgo potencial. La distribución y los nichos ecológicos de T. brasiliensis fueron también caracterizados con este modelado ecológico (30).

En el presente trabajo, investigamos las relaciones entre $T$. dimidiata y los factores bioclimáticos en la península de Yucatán, México, y desarrollamos modelos predictivos de la abundancia de $T$. dimidiata en los domicilios y de su tasa de infección por T. cruzi. Estas predicciones fueron utilizadas para elaborar el primer mapa de riesgo de transmisión natural de la enfermedad de Chagas en la península de Yucatán, una herramienta que puede ser de gran utilidad para el diseño e implementación de un programa de control vectorial en la región.

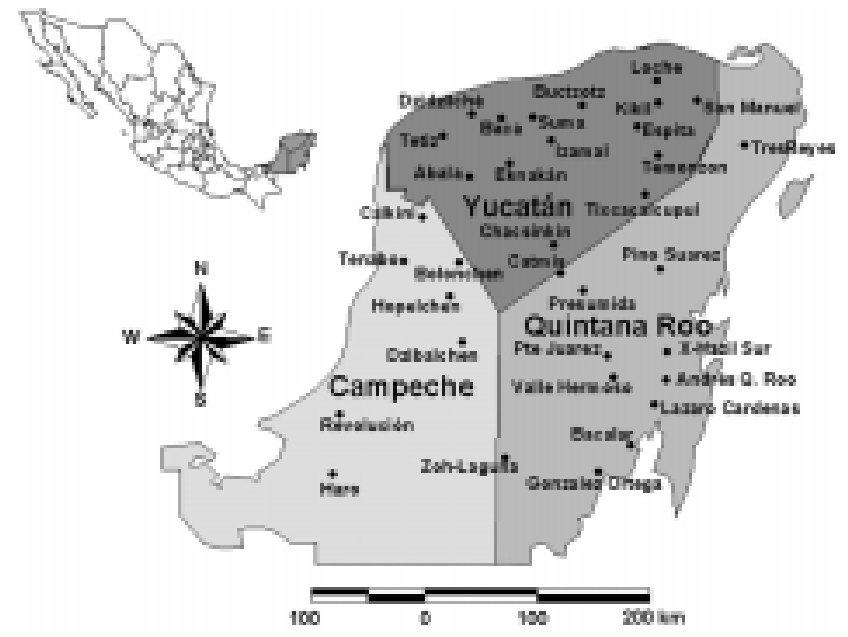

Figura 1.- Mapa del área de estudio. El mapa superior izquierdo representa a México. El mapa principal muestra a la península de Yucatán, con los estados de Campeche, Yucatán, y Quintana Roo. Los círculos indican la ubicación de las localidades donde los datos de campo fueron tomados.

\section{MATERIAL Y MÉTODOS.}

\section{Recolección de datos y procesamiento.}

La península de Yucatán está localizada en el Sureste de México, entre 86--92 ${ }^{\circ}$ de longitud oeste y 17--22 $2^{\circ}$ de latitud norte. Incluye a los estados de Campeche, Yucatán y Quintana Roo.

Los datos entomológicos de 23 localidades de la penínula de Yucatán fueron obtenidos de un estudio de campo anterior (4). Estos incluyen la abundancia de $T$. dimidiata y su tasa de infección por T. cruzi para cada localidad. Además, se colectaron insectos en 11 nuevas localidades durante 2001, y fueron analizados para determinar su tasa de infección por T. cruzi con la misma metodología. Así, utilizamos datos entomológicos de un total de 34 sitios de colecta (Figura 1).

Los mapas digitales de la temperatura anual promedio, la temperatura mínima y máxima, la precipitación pluvial, la humedad relativa, el agua precipitable, la presión atmosférica, y la velocidad del viento correspondientes a los años de los estudios de campo fueron obtenidos del Centro de Diagnostico del Clima NOAA-CIRES, Boulder, Colorado, USA (http://www.cdc.noaa.gov/), que provee variables climáticas modeladas basadas en una combinación de 


\section{E Dumonteil, S Gourbière.}

observaciones de campo, de sensores aéreos y de satélites (31). La precipitación pluvial, las temperaturas minimas, maximas y promedio y la evaporación de un total de 140 estaciones meteorológicas georeferenciadas de la península de Yucatán fueron obtenidas de la Comisión Nacional del Agua (Mérida, Yucatán, México). Los datos demográficos (número de habitantes y de casa de las localidades y municipios) y administrativos fueron obtenidos del Censo Nacional de Población 2000 del INEGI, así como las georeferencias de cada localidad donde se realizaron las colectas. Para el tipo de vegetación, utilizamos una imagen de satélite de Marzo de 2000 del sensor SeaWiFS y realizamos una clasificación supervisada en cuatro grandes tipos de vegetación, correspondiendo a manglares, áreas agrícolas y pastizales, selva mediana y selva alta con el programa Multispec. El valor de Kappa fue mayor a 0.9 para cada tipo de vegetación, indicando una identificación de las clases de vegetación sin ambigüedad. Adicionalmente, la verificación de cada sitio de colecta en el campo confirmó la clasificación de la imagen de satélite.

La localización geográfica de los pacientes seropositivos reportados durante 2003 a través del programa de tamizaje de donadores de sangre fueron proporcionados por el Dr. R. Ojeda-Baranda, (Secretaría de Salud, Mérida, Yucatán), y las localidades de residencia de pacientes con cardiopatía chagásica crónica fueron proporciónadas por el Dr. V. Suárez-Solís (Universidad Autónoma de Yucatán, Mérida, México).

Todos los datos fueron importados en un SIG en modo ráster (MacGIS 3.0, Universidad de Oregon, Eugene, OR, USA) para elaborar una base de datos entomológicos y de factores bioclimáticos de la península de Yucatán. Los datos de las estaciónes meteorológicas fueron interpolados para generar datos continuos para toda el área de estudio. Todos los mapas fueron convertidos a una resolución de $13 \mathrm{x}$ $13 \mathrm{~km}$ por pixel. Los datos bioclimáticos de cada uno de los 34 sitios de colecta fueron extraídos de la base de datos para el análisis estadístico y el modelaje.

\section{Modelaje.}

Para empezar, dividimos los datos en dos conjuntos, uno para ajustar los modelos y uno para evaluarlos. Para esto, los datos fueron apareados para asegurar que rangos similares de abundancia y de tasa de infección, así como datos de los dos años de colecta, fueran representados en ambos conjuntos. Entonces, el modelado del riesgo de transmisión de la enfermedad de Chagas se llevó a cabo en dos etapas. Primero, modelamos la abundancia de $T$. dimidiata en los domicilios en función de las variables bioclimáticas a partir del primer conjunto de datos (17 puntos) con una regresión logística utilizando una distribución de Poisson del error de la probabilidad de ocurrencia de los insectos, con el programa GLIM 3.77. El modelo fue elaborado paso a paso, incorporando las variables una por una en la regresión y para cada paso se conservó únicamente la variable que mejoraba más el ajuste del modelo definido como su varianza. Las variables que no mejoraban el ajuste fueron descartadas del modelo. La abundancia del vector fue entonces ajustada a la ecuación: $A=\exp \left(\beta_{\text {o }}\right.$ $\left.+\beta_{1} X_{1}+\beta_{2} X_{2}+\ldots+\beta_{n} X_{n}\right)$, donde A representa la abundancia, $\beta_{n}$ los coeficientes respectivos de la regresión y $X_{n}$ las respectivas variables incorporadas en el modelo. La relación entre la abundancia ajustada y observada se evaluó por análisis de correlación y regresión lineal. Para evaluar más profundamente el valor predictivo del modelo, lo aplicamos al segundo conjunto de datos (17 puntos) y de la misma manera comparamos la abundancia predicha por el modelo con la abundancia observada por análisis de correlación y regresión lineal.

Segundo, modelamos el número de insectos infectados por T. cruzi por regresión logística con un error binomial de la probabilidad de infección. Como se colectaron insectos en sólo 27 de los 34 sitios del estudio, utilizamos 14 puntos del primer conjunto de datos para ajustar el modelo. El número de insectos infectados se ajustó con la ecuación: $\mathrm{N}_{\mathrm{i}}=\mathrm{N}_{\mathrm{a}}(\mathrm{Y} / 1+$ $\mathrm{Y}$ ), donde $\mathrm{N}_{\mathrm{i}}$ es el numero de insectos infectados, $\mathrm{N}_{\mathrm{a}}$ el número de insectos analizados y $Y=\exp \left(\beta_{0}+\beta_{1} X_{1}\right.$ $\left.+\beta_{2} X_{2}+\ldots+\beta_{n} X_{n}\right)$, con $\beta_{n}$ representando los coeficientes respectivos de la regresión y $X_{n}$ las

\section{Revista Biomédica}


Mapa de riesgo de la enfermedad de Chagas.

respectivas variables del modelo. Como antes, evaluamos la relación entre las tasas de infección ajustadas y observadas por el análisis de correlación y de regresión lineal. También, el valor predictivo del modelo se evaluó con el segundo conjunto de datos (13 puntos) comparando los números predicho y observado de insectos infectados por T. cruzi.

Los valores de los factores bioclimáticos fueron entonces importados de la base de datos del SIG a hojas de cálculo de Excel (Microsoft, USA) y los modelos de regresión logística fueron aplicados a toda el área de estudio. Mapas de la abundancia de $T$. dimidiata y su tasa de infección fueron generados en el SIG. Finalmente, definimos el riesgo de transmisión natural de $T$. cruzi como directamente proporcional al producto de la abundancia de $T$. dimidiata y su tasa de infección por T. cruzi (i.e. proporcional a la abundancia de insectos infectados) y generamos un mapa de riesgo de transmisión. El riesgo se dividió en tres niveles (bajo, mediano y alto), correspondiendo a los tercios más bajo, mediano y más alto de la abundancia de insectos infectados, respectivamente. La validez del mapa de riesgo obtenido fue evaluada comparando la ubicación de casos humanos seropositivos y de cardiopatía chagásica crónica con los niveles de riesgo estimados.

\section{RESULTADOS.}

\section{Modelado de la abundancia de $T$. dimidiata.}

Utilizando el primer conjunto de datos, solamente cinco de todas las variables bioclimáticas y demográficas estudiadas fueron conservadas durante
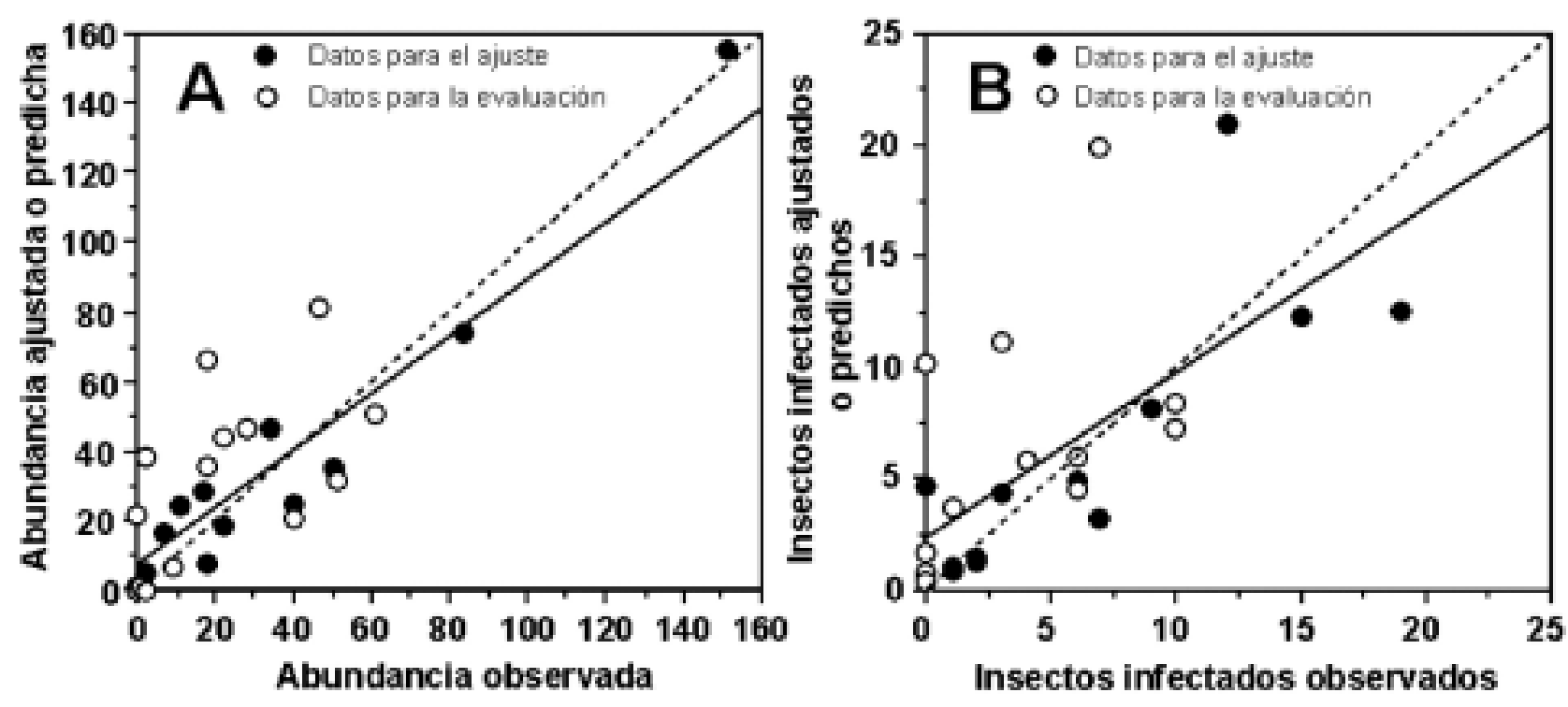

Figura 2.- Relación entre los datos entomológicos observados y ajustados/predichos. (A) Abundancia de T. dimidiata observada y ajustada del conjunto de datos utilizado para la elaboración del modelo (círculos negros), y abundancia de $T$. dimidiata observada y predicha del conjunto de datos utilizados para la evaluación del modelo (círculos blancos). La relación para el conjunto de datos para la elaboración del modelo fue: Abundancia ajustada $=-0.83+1.06$ x Abundancia observada $\left(\mathrm{r}^{2}=0.83, P<0.001\right.$; el último punto fue excluido del análisis de regresión por estar demasiado aislado del resto de los datos). La relación para el conjunto de datos para evaluar el modelo fue: Abundancia predicha $=11.43+0.83 \mathrm{x}$ Abundancia observada $\left(\mathrm{r}^{2}=0.45, P=0.003\right)$, y para la combinación de ambos conjuntos de datos: Abundancia predicha $=$ $7.15+0.82$ x Abundancia observada; línea diagonal $\left(r^{2}=0.59, P<0.001\right)$. La línea diagonal interrumpida indica una relación perfecta. (B) Número de T. dimidiata infectados por T. cruzi observados y ajustados para el conjunto de datos utilizados para elaborar el modelo (círculos negros) y número de $T$. dimidiata infectados por $T$. cruzi observados y predichos para el conjunto de datos utilizados para evaluar el modelo (círculos blancos). La relación entre datos observados y ajustados para el conjunto de datos para elaborar el modelo fue: Infección ajustada $=1.05+0.81$ Infección observada $\left(\mathrm{r}^{2}=0.68, P<0.001\right)$, y para el conjunto de datos para la evaluación del modelo: Infección predicha $=3.55+0.73 \mathrm{x}$ Infección observada, $\left(\mathrm{r}^{2}=0.26\right.$, $P=0.07)$ y para los dos conjuntos de datos combinados: Infección predicha $=2.54+0.73$ x Infección observada; línea diagonal $\left(\mathrm{r}^{2}=0.45, P<0.001\right)$. La línea diagonal interrumpida indica una relación perfecta.

Vol.15/No.4/Octubre-Diciembre, 2004 


\section{E Dumonteil, S Gourbière.}

la elaboración paso a paso de la regresión logística. La velocidad del viento, la precipitación pluvial, el tipo de vegetación, la humedad relativa y la temperatura máxima permitieron reducir la varianza del modelo de 744.5 hasta 58.1 (Grados de libertad $(\mathrm{gl})=10)$. La correlación entre la abundancia ajustada y observada fue fuerte para los 17 sitios utilizados para elaborar el modelo, y la pendiente de la regresión se acercó a 1 (Fig. 2A, $\mathrm{r}^{2}=0.55, P<0.001$; pendiente $=1.06 \pm 0.12$ ). Por otro lado, los residuos de la regresión logística no presentaron correlación con la abundancia observada, ni con las variables dependientes o con la abundancia ajustada (datos no mostrados). Evaluamos entonces el valor predictivo del modelo con el segundo conjunto de datos. Nuevamente, hubo una correlación fuerte entre la abundancia predicha y observada, y la pendiente de la regresión se acerco a 1 (Fig. $2 \mathrm{~A} ; \mathrm{r}^{2}=0.45, P=$ 0.003 ; slope $=0.83 \pm 0.24)$. Globalmente, la combinación de los dos conjuntos de datos indicó una buena coincidencia entre la abundancia de $T$. dimidiata predicha y observada (Abundancia predicha $=7.15+0.82 \times$ Abundancia observada; $\mathrm{r}^{2}$ $=0.59, P<0.001)$.

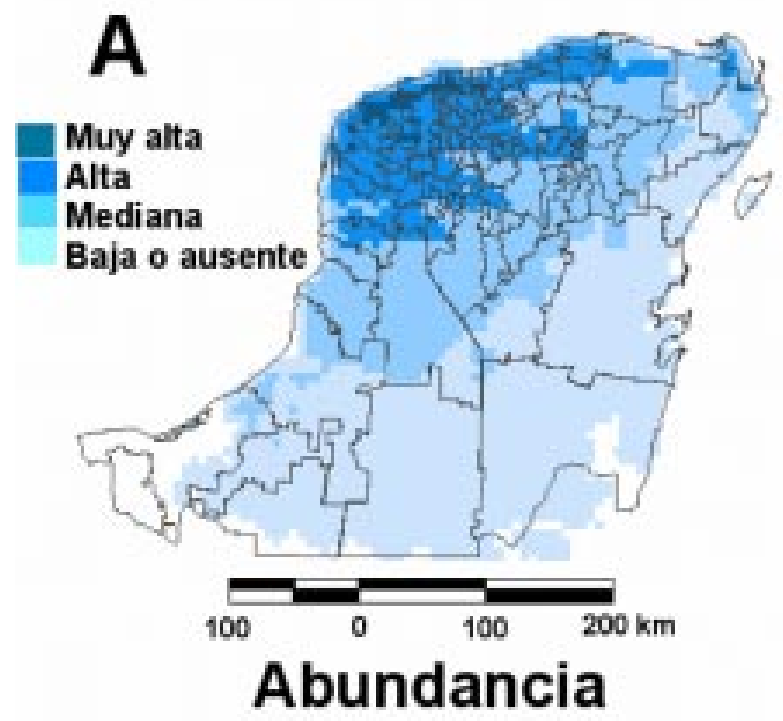

Modelado de la infección de $\boldsymbol{T}$. dimidiata por $\boldsymbol{T}$. cruzi.

De los 27 sitios en los cuales se colectaron $T$. dimidiata, utilizamos 14 sitios para ajustar el número de insectos infectados con las variables bioclimáticas y demográficas. La humedad relativa, la precipitación pluvial, y las temperaturas mínimas y máximas permitieron reducir la varianza de 55.1 a $35.9(\mathrm{gl}=7)$. Hubo una buena correlación entre los números ajustados y observados de insectos infectados para estos 14 sitios (Fig. 2B, $\mathrm{r}^{2}=0.68, p<0.001$; pendiente $=0.81 \pm 0.16)$. Nuevamente, los residuos no presentaron correlación con las tasas de infección ni con las otras variables (datos no mostrados). Cuando evaluamos el valor predictivo del modelo con el segundo conjunto de 13 sitios, observamos una baja correlación entre los números predichos y observados de insectos infectados (Fig. 2B; $r^{2}=0.26, P=0.07$; pendiente $=0.73 \pm 0.36$ ). Sin embargo, la combinación de los dos conjuntos de datos indicó una buena coincidencia entre los números predichos y observados de insectos infectados (Infección predicha $=2.54+$ 0.73 x Infección observada; $\mathrm{r}^{2}=0.45, P<0.001$ ).

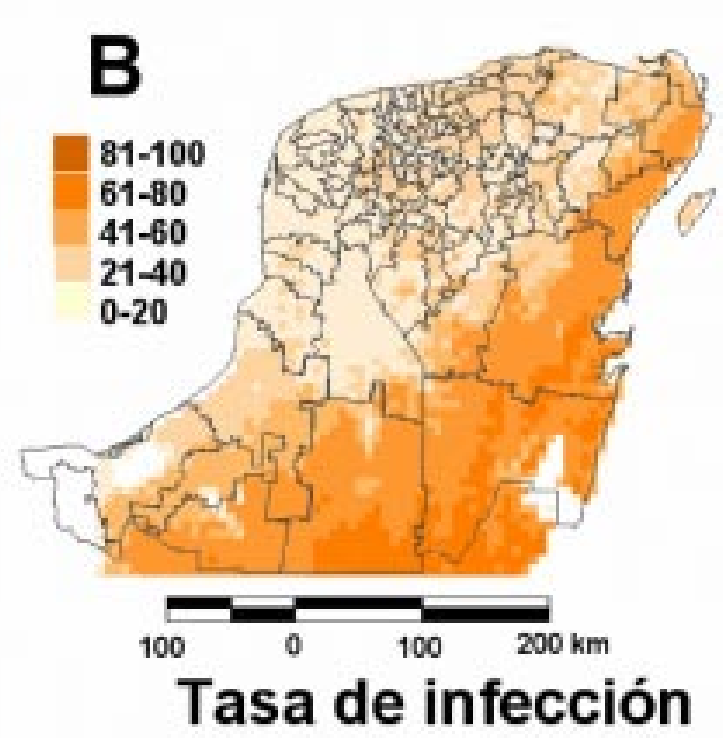

Figura 3.- Mapas de predicciones de la abundancia de T. dimidiata y su infección por T. cruzi. Los modelos predictivos de la abundancia doméstica de T. dimidiata (A) y de su tasa de infección por T. cruzi (B) fueron aplicados a toda el área de estudio. La abundancia fue dividida en cuatro niveles arbitrarios y codificados por colores desde nula o baja hasta muy alta, y la infección fue similarmente dividida en 5 intervalos cubriendo de 0 hasta 100\% de infección. Las líneas representan los límites administrativos de los municipios de los diferentes estados. 
Mapa de riesgo de la enfermedad de Chagas.

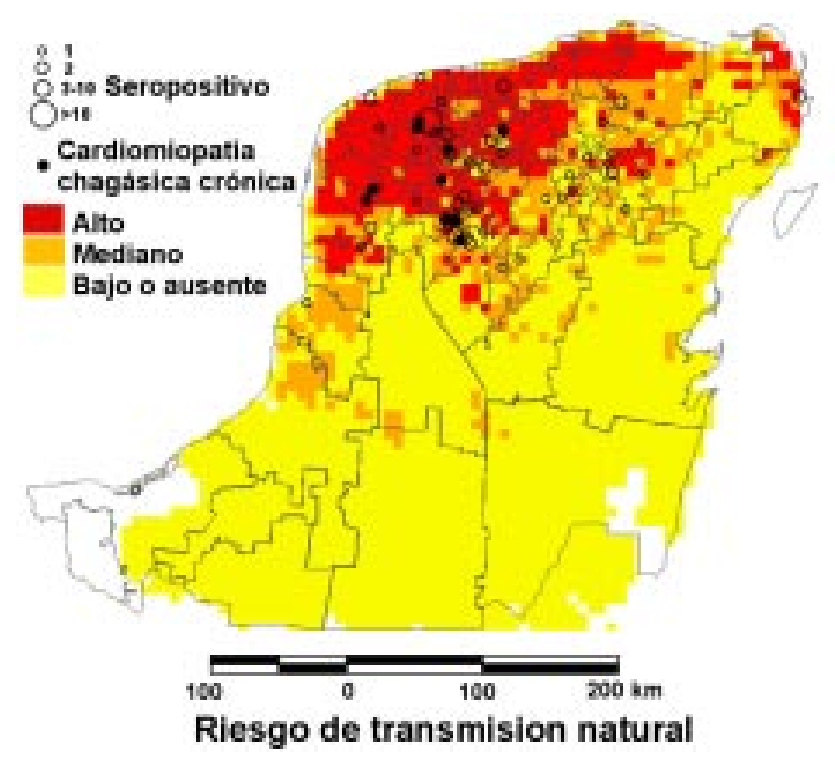

Figura 4.- Mapa de riesgo de transmisión natural de la enfermedad de Chagas. El riesgo de transmisión natural de la enfermedad de Chagas, definido como proporcional a la abundancia de $T$. dimidiata infectados y obtenido de la combinación de los mapas Fig 3A y 3B, fue dividido en tres categorias codificadas por colores, desde riesgo ausente o bajo hasta alto. Los círculos vacíos y negros indican la ubicación de casos seropositivos y de cardiopatía chagásica crónica, respectivamente, con el tamaño del círculo vacío proporcional al número de casos como se indica.

\section{Mapa de riesgo de transmisión natural.}

Aplicamos entonces ambos modelos a toda el área de estudio para obtener mapas de la abundancia doméstica de T. dimidiata y de su tasa de infección por T. cruzi en la península de Yucatán (Fig. 3A y 3B, respectivamente). Como se esperaba, y de acuerdo con nuestro estudio anterior (4), estos mapas indicaron una distribución geográfica muy heterógenea de la abundancia de T. dimidiata, siendo muy abundante en el noroeste de la península, y más escasa en la parte sur. De la misma manera, las tasas de infección variaron mucho, siendo más baja en el noroeste, y más alta en el sureste. Definimos entonces el riesgo de transmisión como directamente proporciónal a la abundancia de insectos infectados, que se obtuvo combinando los mapas de abundancia de T. dimidiata y de su tasa de infección por $T$. cruzi (Fig. 4). El riesgo de transmisión natural resultó alto en la mayor parte del estado de Yucatán, así como en el norte de los estados de Campeche y de Quintana Roo. Por otra parte, el riesgo de transmisión resultó bajo en el sur de Campeche y de Quintana Roo. Para probar aún mas el valor predictivo de este mapa de riesgo, comparamos la ubicación geográfica de casos humanos seropositivos para T. cruzi y de casos de cardiopatía chagásica crónica con el riesgo de transmisión predicho. Como se indica en las figuras 4 y 5, la mayoría de los pacientes seropositivos venían de localidades con un alto riesgo de transmisión predicho, y solamente 7 pacientes fueron de un área de bajo riesgo de transmisión. Similarmente, 9/10 pacientes con cardiopatía chagásica crónica fueron de áreas de alto riesgo, 1/10 de áreas de riesgo mediano, y ningúno de áreas de bajo riesgo, confirmando el buen valor predictivo del mapa de riesgo de transmisión.

\section{DISCUSIÓN.}

Como otras enfermedades transmitidas por vectores, la enfermedad de Chagas es fuertemente influenciada por factores ambientales y bioclimáticos, y numerosos estudios han investigado el efecto de

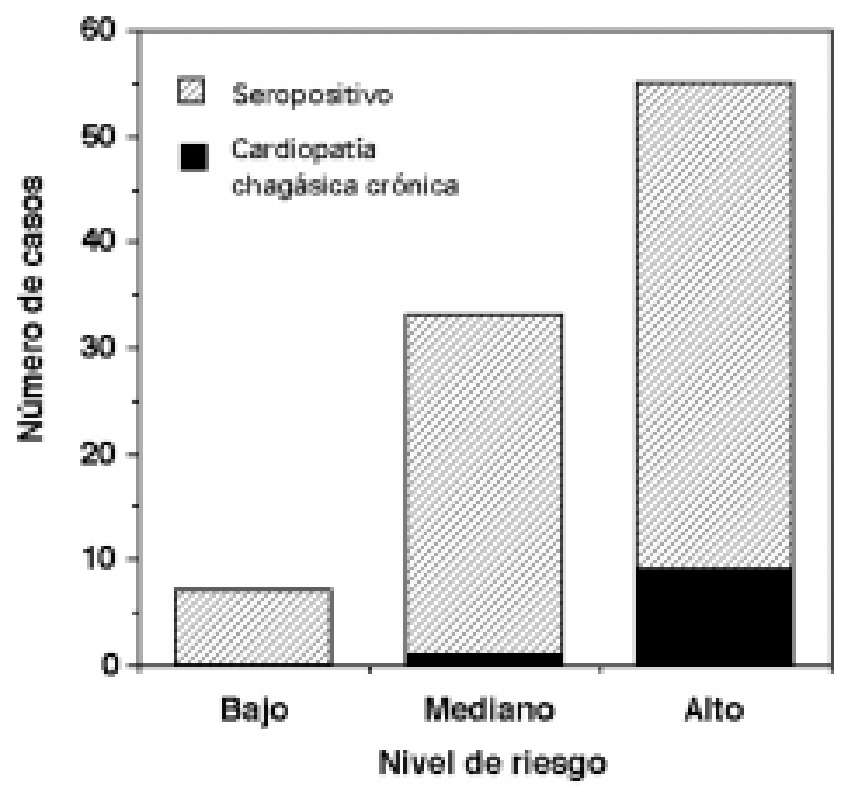

Figura 5.- Relación entre el nivel de riesgo y el número de casos seropositivos y de cardiopatía chagásica crónica. El número de pacientes seropositivos (zona rayada) y pacientes con cardiopatía chagásica crónica (zona negra) se muestran para cada nivel de riesgo predicho. 


\section{E Dumonteil, S Gourbière.}

factores climáticos sobre varios aspectos del ciclo de vida de los triatomineos (8). Sin embargo, muy pocos estudios han intentado integrar las relaciones entre las variables ambientales y los vectores de la enfermedad de Chagas para predecir el riesgo de transmisión (5). En este estudio, presentamos el primer mapa de riesgo de transmisión de la enfermedad de Chagas en la península de Yucatán, México. Este mapa puede ser de gran utilidad para futuros estudios epidemiológicos y entomológicos, ya que permitirá enfocar estos estudios en las áreas de mayor riesgo. Pero aún más importante, este mapa representa una herramienta muy importante de ayuda a la toma de decisiones para programas de control vectorial y el seguimiento epidemiológico en la región, permitiendo optimizar el costo-beneficio de estos programas de salud pública.

Nuestros modelos permiten la predicción de la abundancia de $T$. dimidiata en los domicilios y de su infección por T. cruzi, y encontramos una buena correpondencia entre los datos predichos y observados, tanto para aquellos utilizados para elaborar los modelos como los utlizados para probarlos. La predicción de la abundancia del vector fue muy precisa, con más de $90 \%$ de la variación explicada por el modelo. De las variables incorporadas en este modelo, la influencia de la temperatura y de la precipitación pluvial/humedad sobre la biologia y el ciclo de vida de los triatomineos ha sido bien establecida para varias especies (8) y $T$. dimidiata fue más abundante en la península de Yucatán donde el clima es más caluroso y seco. Esto contrasta con las poblaciones T. dimidiata de Oaxaca, México, que están restringidas a áreas más templadas (5). Así, es posible que existan importantes diferencias entre los nichos ecológicos de estas poblaciónes de $T$. dimidiata, lo que confirma las también importantes diferencias genéticas observadas entre ellas (32). De hecho, se ha sugerido que las poblaciónes de $T$. dimidiata de la península de Yucatán se encuentran en un fuerte proceso de diferenciación (32), que puede manifestarse en diferencias biológicas y ecológicas muy marcadas con otras poblaciónes de México y Centroamerica.

De manera interesante, encontramos que la velocidad del viento influyó fuertemente en la abundancia doméstica de $T$. dimidiata, lo que puede ser interpretado como el reflejo de un evento de dispersión. De acuerdo con esta interpretación, sugerimos en un estudio anterior que la infestación de las casas por $T$. dimidiata en la región, se debía sobre todo a la dispersión estacional de insectos adultos silvestres o peridomiciliares (4), nuevamente en contraste con otras poblaciones de T. dimidiata que presentan una domiciliación más fuerte (5, 33-35). La incorporación de las variaciones estacionales en futuros estudios de mapeo del riesgo podría ayudar a identificar los posibles factores bioclimáticos relacionados con la infestación estacional de los domicilios en la península de Yucatán.

La precisión del modelo de predicción de la infección de T. dimidiata por T. cruzi fue más baja, con solamente $35 \%$ de la variación explicada por el modelo. Sin embargo, hubo suficiente concordancia entre la infección de $T$. dimidiata predicha y observada de acuerdo con el análisis de regresión y correlación. El menor ajuste del modelo se puede explicar, en parte, por el número más reducido de puntos disponibles para elaborar este modelo, pero también y más probablemente porque no se incluyeron en nuestro estudio factores que influyen de manera más importante sobre la infección de $T$. dimidiata por T. cruzi. Se ha demostrado en otro estudio que las variables relacionadas con la temperatura y la humedad pueden directamente modular el desarrollo de $T$. cruzi en $T$. infestans (36), y estas variables fueron incorporadas en nuestro modelo. Una infección más alta fue asociada a una temperatura más baja y una humedad/precipitación pluvial más alta. Sin embargo, es muy probable que la infección dependa más criticamente de varios factores adicionales como, por ejemplo, las especies de hospederos sobre las cuales los insectos se alimentan y sus respectivas tasas de infección por T. cruzi, pero estos datos todavía no se conocen. La falta de conocimientos relacionados con estos aspectos representa una limitación importante para la elaboración de mapas más confiables de la infección de los vectores (37).

El tipo de vegetación fue también un predictor 


\section{Mapa de riesgo de la enfermedad de Chagas.}

importante tanto de la abundancia como de la infección de $T$. dimidiata. Este es en realidad un indicador que caracteriza de manera muy general el ambiente, ya que integra un gran número de factores climáticos, geológicos, zoológicos y antropológicos, y es de hecho el factor más frecuentemente asociado a la distribución geográfica de vectores $(13,20,23,28,38)$. Esto implica que el uso de datos más detallados sobre la vegetación como los datos de NDVI obtenidos por percepción remota pudieran pemitir la elaboración de modelos más sencillos, pero posiblemente con mayor resolución espacial. La mayor abundancia de insectos se asoció a la vegetación perturbada por actividades humanas (agricultura y pastizales), lo que sugiere que la desforestación y la degradación de habitats son factores que contribuyen a la infestación de las casas por $T$. dimidiata. Por otra parte, altos niveles de infección por $T$. cruzi coincidieron con áreas de selvas altas, en concordancia con el origen silvestre y zoonótico de los parásitos.

Nuestro mapa de riesgo de transmisión natural fue de alto valor predictivo para los casos humanos. La gran mayoría de los pacientes detectados en años recientes vinieron de las áreas de alto riesgo, mientras que solamente dos fueron de áreas predichas de bajo riesgo. La abundancia de vectores infectados en los domicilios es el indicador de transmisión natural más directo, pero factores adicionales como la presencia de animales domésticos pueden modular el riesgo de transmisión a humanos (39). Nuestro mapa predice que en la península de Yucatán, alrededor de 1.6-1.7 millones de personas están expuestas a un riesgo de transmisión de T. cruzi mediano-alto, con más de $90 \%$ de éstas localizadas en el estado de Yucatán, y 1.51.6 millones de personas viven en áreas de bajo riesgo. Este mapa representa una primera herramienta de ayuda a la toma de decisiones por parte de las autoridades de salud para la identificación de áreas prioritarias para el control vectorial. Estudios adicionales y refinamientos de este tipo de análisis podrían permitir la elaboración de mapas de mayor resolución espacial y mayor valor predictivo. El uso generalizado de herramientas de este tipo para el control vectorial de la enfermedad de Chagas y la vigilancia epidemiológica permitiría optimizar estos programas y una mejor distribución de los recursos.

\section{AGRADECIMIENTOS.}

Este trabajo fue financiado por el convenio \#20020404 de SISIERRA/CONACYT a E.D.. Los autores agradecen a los Drs. R. Ojeda-Baranda y V. Suarez-Solis por los datos clínicos y a los Drs. J. Brownstein y J.A. Farfán-Ale por discusiones y comentarios en la elaboración de este trabajo. Agradecemos también a la Dra. G. Sánchez-Burgos por la revisión del texto en español.

\section{REFERENCIAS.}

1.- WHO. Chagas disease. Progress towards eliminatión of transmissión. Wkly Epidemiol Rec 1996; 71:12-5.

2.- Dumonteil E. Update on Chagas' disease in Mexico. Salud Publica Mex 1999;41:322-7.

3.- WHO. Chagas disease: Central American initiative launched. TDR News 1998;6.

4.- Dumonteil E, Gourbiere S, Barrera-Perez M, RodriguezFelix E, Ruiz-Piña H, Baños-Lopez O, Ramirez-Sierra MJ, Menu F, Rabinovich JE. Geographic distribution of Triatoma dimidiata and transmission dynamics of Trypanosoma cruzi in the Yucatán península of Mexico. Am J Trop Med Hyg 2002; 67:176-83.

5.- Ramsey JM, Ordonez R, Cruz-Celis A, Alvear AL, Chavez V, Lopez R, Pintor JR, Gama F, Carrillo S. Distribution of domestic triatominae and stratification of Chagas Disease transmission in Oaxaca, Mexico. Med Vet Entomol 2000; 14:19-30.

6.- Zeledon R, Ugalde JA, Paniagua LA. Entomological and ecological aspects of six sylvatic species of triatomines (Hemiptera, Reduviidae) from the collection of the National Biodiversity Institute of Costa Rica, Central America. Mem Inst Oswaldo Cruz 2001; 96: 757-64.

7.- Galvao C, Jurberg J, Carcavallo RU, Segura CA, Galindez Giron I, Curto de Casas SI. Distribuição geográfica e dispersão alti-latitudinal de aluns gêneros e espécies da tribo Triatomini Jeannel, 1919 (Hemiptera, Reduviidae, Triatominae). Mem Inst Oswaldo Cruz 1998; 93:33-7.

8.- Carcavallo RU,. Climatic factors related to Chagas disease transmissión. Mem Inst Oswaldo Cruz 1999; 94 Suppl 1:3679. 


\section{E Dumonteil, S Gourbière.}

9.- Rejmankova E, Roberts DR, Pawley A, Manguin S, Polanco J. Predictión of Anopheles albimanus densities in villages based on distances to remotely sensed larval habitats. Am J Trop Med Hyg 1995; 53:482-8.

10.- Rogers DJ, Randolph SE, Snow RW, Hay SI,. Satellite imagery in the study and forecast of malaria. Nature 2002; 415:710-5.

11.- Snow RW, Craig MH, Deichmann U, le Sueur D,. A preliminary continental risk map for malaria mortality among African children. Parasitol Today 1999; 15:99-104.

12.- Beck LR, Rodriguez MH, Dister SW, Rodriguez AD, Rejmankova E, Ulloa A, et al. Remote sensing as a landscape epidemiologic tool to identify villages at high risk for malaria transmissión. Am J Trop Med Hyg 1994; 51:271-80.

13.- Thomson MC, Connor SJ, D'Alessandro U, Rowlingson Z, Diggle P, Cresswell M, et al. Predicting malaria infection on Gambian children from satellite data and bed net use surveys: the importance of spacial correlation in the interpretation of the results. Am J Trop Med Hyg 1999; 61:28.

14.- Rogers DJ, Randolph SE. Mortality rates and population density of tsetse flies correlated with satellite imagery. Nature 1991;351:739-41.

15.- Rogers DJ, Williams BG. Monitoring trypanosomiasis in space and time. Parasitology 1993;106 Suppl: S77-S92.

16.- Brooker S, Hay SI, Bundy DA. Tools from ecology: useful for evaluating infection risk models? Trends Parasitol 2002; 18:70-4.

17.- Kristensen TK, Malone JB, McCarroll JC. Use of satellite remote sensing and geographic information systems to model the distribution and abundance of snail intermediate hosts in Africa: a preliminary model for Biomphalaria pfeifferi in Ethiopia. Acta Trop 2001; 79:73-8.

18.- Elnaiem DA, Schorscher J, Bendall A, Obsomer V, Osman ME, Mekkawi AM, et al. Risk mapping of visceral leishmaniasis: the role of local variation in rainfall and altitude on the presence and incidence of kala-azar in eastern Sudan. Am J Trop Med Hyg 2003; 68:10-7.

19.- Elnaiem DA, Connor SJ, Thomson MC, Hassan MM, Hassan HK, Aboud MA, et al. Environmental determinants of the distribution of Phlebotomus orientalis in Sudan. Ann Trop Med Parasitol 1998; 92:877-87.

\section{Revista Biomédica}

20.- Cross ER, Newcomb WW, Tucker CJ. Use of weather data and remote sensing to predict the geographic and seasonal distributión of Phlebotomus papatasi in southwest Asia. Am J Trop Med Hyg 1996; 54:530-6.

21.- Thomson MC, Elnaiem DA, Ashford RW, Connor SJ. Towards a kala azar risk map for Sudan: mapping the potential distribution of Phlebotomus orientalis using digital data of environmental variables. Trop Med Int Health 1999; 4:10513.

22.- Kitron U. Landscape ecology and epidemiology of vector-borne diseases: tools for spatial analysis. J Med Entomol 1998; 35:435-45.

23.- Guerra M, Walker E, Jones C, Paskewitz S, Cortinas MR, Stancil A, et al. Predicting the risk of Lyme disease: habitat suitability for Ixodes scapularis in the north central United States. Emerg Infect Dis 2002; 8:289-97.

24.- Brownstein JS, Holford R, Fish D. A climate-based model predicts the spatial distribution of the Lyme disease vector Ixodes scapularis in the United States. Environ Health Perspect 2003; 111:1152-7.

25.- Malone JB, Bergquist NR, Huh OK, Bavia ME, Bernardi M, El Bahy MM, et al. A global network for the control of snail-borne disease using satellite surveillance and geographic information systems. Acta Trop 2001; 79:7-12.

26.- Clark JS, Carpenter SR, Barber M, Collins S, Dobson A, Foley JA, et al. Ecological forecasts: an emerging imperative. Science 2001; 293:657-60.

27.- Rushton G. Public health, GIS, and spatial analitic tools. Annu Rev Public Health 2003; 24:43-56.

28.- Gorla DE. Variables ambientales registradas por sensores remotos como indicadores de la distribución geográfica de Triatoma infestans. Ecología Austral 2002; 12:117-27.

29.- Peterson AT, Sanchez-Cordero V, Beard CB, Ramsey JM. Ecologic niche modeling and potential reservoirs for Chagas disease, Mexico. Emerg Infect Dis 2002; 8:662-7.

30.- Costa J, Peterson AT, Beard CB. Ecologic niche modeling and differentiation of populations of Triatoma brasiliensis neiva, 1911, the most important Chagas' disease vector in northeastern Brazil (hemiptera, reduviidae, triatominae). Am J Trop Med Hyg 2002; 67:516-20.

31.- Kalnay E, Kanamitsu M, Kistler R, Collins W, Deaven D, Gandin L, et al. The NCEP/NCAR 40 year reanalysis project. 
Bull Amer Meteor Soc 1996-77:437-71.

32.- Bargues MD, Marcellina A, Dujardin JP, Más-Coma S. Triatomine vector of Trypanosoma cruzi: a molecular perspective based on nuclear ribosomal DNA markers. Trans R Soc Trop Med Hyg 2002; 96 (Suppl 1):159-64.

33.- Zeledón R. El Triatoma dimidiata (Latreille, 1811) y su relación con la enfermedad de Chagas. San José (Costa Rica): UNED, 1981.

34.- Zeledon R, Montenegro VR, Zeledon O. Evidence of colonization of man-made ecotopes by Triatoma dimidiata (Latreille, 1811) in Costa Rica. Mem Inst Oswaldo Cruz 2001; 96:659-60.

35.- Monroy C, Rodas A, Mejia M, Rosales R, Tabaru Y. Epidemiology of Chagas disease in Guatemala: infection rate of Triatoma dimidiata, Triatoma nitida and Rhodnius prolixus (Hemiptera, Reduviidae) with Trypanosoma cruzi and Trypanosoma rangeli (Kinetoplastida, Trypanosomatidae). Mem Inst Oswaldo Cruz 2003; 98:305-10.

36.- Asin S, Catala S. Development of Trypanosoma cruzi in Triatoma infestans: influence of temperature and blood consumption. J Parasitol 1995; 81:1-7.

37.- Randolph SE. Ticks and tick-borne disease systems in space and from space. Adv Parasitol 2000; 47:217-45.

38.- Estrada-Peña A. Distribution, abundance, and habitat preferences of Ixodes ricinus (Acari: Ixodidae) in northern Spain. J Med Entomol 2001; 38:361-70.

39.- Cohen J, Gürtler RE. Modeling household transmission of american trypanosomiasis. Science 2001; 293:694-8. 